\title{
ADAPTIVE DETERMINISTIC DYADIC GRIDS ON SPACES OF HOMOGENEOUS TYPE
}

\author{
RICHARD LECHNER AND MARKUS PASSENBRUNNER
}

\begin{abstract}
In the context of spaces of homogeneous type, we develop a method to deterministically construct dyadic grids, specifically adapted to a given combinatorial situation. This method is used to estimate vector-valued operators rearranging martingale difference sequences such as the Haar system.
\end{abstract}

\section{INTRODUCTION}

In [5, 6], T. Figiel developed martingale methods to prove a vector-valued $T(1)$ theorem by decomposing the singular integral operator $T$ into an absolutely converging series of basic building blocks $T_{m}$ and $U_{m}, m \in \mathbb{Z}$. Those operators are given by the linear extension of

$$
T_{m} h_{I}=h_{I+m|I|} \quad \text { and } \quad U_{m} h_{I}=\mathbb{1}_{I+m|I|}-\mathbb{1}_{I},
$$

where $\left\{h_{I}\right\}$ denotes the Haar system on standard dyadic intervals $I$ and $\mathbb{1}_{A}$ the characteristic function of the set $A$. The crucial norm estimates obtained in [5] take the form

$$
\begin{gathered}
\left\|T_{m}: L_{E}^{p} \rightarrow L_{E}^{p}\right\| \leq C\left(\log _{2}(2+|m|)\right)^{\alpha}, \\
\left\|U_{m}: L_{E}^{p} \rightarrow L_{E}^{p}\right\| \leq C\left(\log _{2}(2+|m|)\right)^{\beta},
\end{gathered}
$$

where $1<p<\infty$ and the constant $C>0$ depends only on $p$, the UMD-constant of the Banach space $E$ and $\alpha, \beta<1$. Estimates (1.2) and (1.3) are obtained by hard combinatorial arguments, analyzing structure and position of dyadic intervals.

T. Figiel's decomposition method was extended in [13] to spaces of homogeneous type to obtain a vector-valued $T(1)$ theorem, requiring norm estimates for the building blocks $T_{m}$ and $U_{m}$ in the setting of spaces of homogeneous type. These estimates are proved by hard combinatorial arguments similar to [5].

In [10, an alternative proof for the estimates of $T_{m}$ and $U_{m}$ is given which eliminates the hard combinatorics in [5] to a great extent. Adapting the dyadic grid by means of an algebraic shift, $T_{m}$ and $U_{m}$ are decomposed into roughly $\log _{2}(2+|m|)$ martingale transform operators, thereby yielding (1.2) and (1.3). The shift of the dyadic grid is accomplished by the one-third-trick, which originates in the work of [4], [17], [7], and [1].

Adaptive dyadic grids also proved to be a valuable tool for estimating so called stripe operators in [9]. Those stripe operators were used in [11] as well as in [9] to show weak lower semi-continuity of functionals with separately convex integrands on scalar-valued $L^{p}$ and vector-valued $L^{p}$, respectively. For the scalar-valued $L^{2}$ version of this result, cf. [14].

In this paper we extend the method from [10] to construct adapted dyadic grids in spaces of homogeneous type, which allow us to

Date: February 27, 2018.

2010 Mathematics Subject Classification. 46E40.

Key words and phrases. Space of homogeneous type, vector-valued, UMD, adaptive dyadic grid, rearrangement operator, stripe operator, martingale difference sequence. 
(i) simplify the combinatorial arguments for the estimation of the rearrangement operators $T_{m}$ used in the proof of the $T(1)$ theorem in [13,

(ii) generalize the vector-valued result in [9] on stripe operators to spaces of homogeneous type.

Related recent developments. In [8], T. P. Hytönen presented a proof of T. Figiel's vector-valued $T(1)$ theorem, cf. [6], based upon randomized dyadic grids, originating in [15, 16]. By contrast, the method developed in the present paper allows us to adapt a dyadic grid deterministically to a given combinatorial situation.

\section{Preliminaries}

In this section we present some basic facts concerning spaces of homogeneous type. For basic facts on UMD-spaces used within this work, the notion of Rademacher type and cotype as well as Kahane's contraction principle and Bourgain's version of Stein's martingale inequality, we refer to [10].

Let $X$ be a set. A mapping $d: X \times X \rightarrow \mathbb{R}_{0}^{+}$with the properties that for all $x, y, z \in X$,

(1) $d(x, y)=0 \Leftrightarrow x=y$,

(2) $d(x, y)=d(y, x)$,

(3) $d(x, y) \leq K_{X}(d(x, z)+d(z, y))$ for some constant $K_{X} \geq 1$ only depending on $X$,

is called a quasimetric and $(X, d)$ is called a quasimetric space. Given a quasimetric $d$, we define the ball centered at $x \in X$ with radius $r>0$ as

$$
B(x, r):=\{y \in X: d(x, y)<r\} .
$$

Additionally, a set $A \subset X$ is called open, if for all $x \in X$ there exists $r>0$ such that $B(x, r) \subseteq A$. Furthermore, for an arbitrary set $A \subset X$ and $r>0$, define

$$
B(A, r):=\{y \in X: d(A, y)<r\} .
$$

Let $(X, d)$ be a quasimetric space such that every ball in the quasimetric $d$ is open and $|\cdot|$ be a Borel measure. If the doubling condition holds, i.e. there is a constant $C_{d}>0$ such that

$$
0<|B(x, 2 r)| \leq C_{d}|B(x, r)|<\infty, \quad x \in X, r>0,
$$

then $(X, d,|\cdot|)$ is called a space of homogeneous type. Since for a given quasimetric space $(X, d)$, the balls in $X$ are not necessarily open, we added this condition to the definition. This is the case, if for instance one imposes a Hölder condition on $d$ : There exists $c<\infty$ and $0<\beta<1$ such that for all $x, y, z \in X$ we have

$$
|d(x, z)-d(y, z)| \leq c \cdot d(x, y)^{\beta} \max \{d(x, z), d(y, z)\}^{1-\beta} .
$$

In fact, R. A. Macías and C. Segovia proved in 12 that for every space of homogeneous type there exists an equivalent quasimetric with the desired Hölder property. Here, a quasimetric $d^{\prime}$ is equivalent to a quasimetric $d$ if there exists a finite constant $c$ such that

$$
\frac{1}{c} d(x, y) \leq d^{\prime}(x, y) \leq c d(x, y), \quad x, y \in X .
$$

Let $\mathscr{C}$ be a collection of arbitrary sets. $\mathscr{C}$ is called nested, if $A \cap B \in\{A, B, \emptyset\}$ for all $A, B \in \mathscr{C}$. Furthermore, for such a given nested collection $\mathscr{C}$ we define the predecessor $\pi_{\mathscr{C}}(C)$ of $C$ with respect to the collection $\mathscr{C}$ by

$$
\pi_{\mathscr{C}}(C):=\bigcap\{D: D \supsetneq C, D \in \mathscr{C} \cup\{X\}\} .
$$


Dyadic cubes. In spaces of homogeneous type, one can construct a collection of subsets that has similar properties to dyadic cubes in $\mathbb{R}^{k}$, cf. M. Christ [2] and G. David [3].

Theorem 2.1. Let $(X, d,|\cdot|)$ be a space of homogeneous type. Then there exists a system $\mathscr{Q}$ of open subsets of $X$ with centers $m_{A} \in A$ of $A \in \mathscr{Q}$ and a splitting $\mathscr{Q}=\cup_{n \in \mathbb{Z}} \mathscr{Q}_{n}$ such that the following properties are satisfied with uniform constants $q<1, C_{1}, C_{2}, C_{3}, \eta \in$ $\mathbb{R}^{+}, N \in \mathbb{N}$ :

(1) For all $n \in \mathbb{Z}$ we have that $X=\bigcup_{A \in \mathscr{Q}_{n}} A$ up to $|\cdot|$-null sets.

(2) For $A \in \mathscr{Q}_{k}$ and $B \in \mathscr{Q}_{n}$ with $k \leq n$, we have either $B \subset A$ or $A \cap B=\emptyset$.

(3) For each $B \in \mathscr{Q}_{n}$ and every $k \leq n$, there is exactly one $A \in \mathscr{Q}_{k}$ such that $B \subset A$.

(4) For all $n \in \mathbb{Z}$ and $A \in \mathscr{Q}_{n}$ we have that $B\left(m_{A}, C_{1} q^{n}\right) \subseteq A \subseteq B\left(m_{A}, C_{2} q^{n}\right)$.

(5) Let $A \in \mathscr{Q}_{n}$. The boundary layer of $A$ having width $t$ is given by

$$
\partial_{t} A:=\left\{x \in A: d(x, X \backslash A) \leq t q^{n}\right\},
$$

and satisfies the measure estimate

$$
\left|\partial_{t} A\right|<C_{3} t^{\eta}|A|
$$

(6) For all $n \in \mathbb{Z}$, the collection $\mathscr{Q}_{n}$ is countable.

(7) For all $n \in \mathbb{Z}$ and $A \in \mathscr{Q}_{n}$ we have $N(A):=\left|\left\{B \in \mathscr{Q}_{n+1}: B \subseteq A\right\}\right| \leq N$.

(8) For all $n \in \mathbb{Z}$ and $A \in \mathscr{Q}_{n}$ there exists a subcollection $\mathscr{S}$ of $\overline{\mathscr{Q}}_{n+1}$ with $|\mathscr{S}| \leq N$ and

$$
A=\bigcup_{B \in \mathscr{S}} B \quad \text { up to }|\cdot| \text {-null sets. }
$$

We define the level of a cube $A \in \mathscr{Q}_{n}$ as lev $A:=n$, and furthermore

$$
r \diamond A:=B\left(A, r q^{\operatorname{lev} A}\right), \quad A \in \mathscr{Q}, r>0 .
$$

In the following, $(X, d,|\cdot|)$ denotes a space of homogeneous type, equipped with a quasimetric $d$ and a measure $|\cdot|$.

Lemma 2.2. Let $A \in \mathscr{Q}$ and $r>0$. Then

$$
r \diamond A \subset B\left(m_{A}, K_{X}\left(C_{2}+r\right) q^{\operatorname{lev} A}\right) .
$$

Proof. Let $z \in r \diamond A=B\left(A, r q^{\operatorname{lev} A}\right)$ and estimate

$$
\begin{aligned}
d\left(m_{A}, z\right) & \leq \inf _{y \in A} K_{X}\left(d\left(m_{A}, y\right)+d(y, z)\right) \\
& \leq K_{X}\left(C_{2} q^{\operatorname{lev} A}+d(A, z)\right) \\
& \leq K_{X}\left(C_{2} q^{\operatorname{lev} A}+r q^{\operatorname{lev} A}\right) .
\end{aligned}
$$

Lemma 2.3. Let $A_{1}, A_{2} \in \mathscr{Q}$ and assume that

$$
\left(r_{1} \diamond A_{1}\right) \cap\left(r_{2} \diamond A_{2}\right) \neq \emptyset
$$

for some $r_{1}, r_{2}>0$. Then

where $r=2 K_{X}^{3}\left(C_{2}+r_{2}\right) q^{\operatorname{lev} A_{2}-\operatorname{lev} A_{1}}+K_{X} r_{1}$.

$$
r_{2} \diamond A_{2} \subset r \diamond A_{1}
$$

Proof. Let $y \in\left(r_{1} \diamond A_{1}\right) \cap\left(r_{2} \diamond A_{2}\right)$ and $z \in\left(r_{2} \diamond A_{2}\right)$, then

$$
d\left(z, A_{1}\right) \leq K_{X}\left(d(z, y)+d\left(y, A_{1}\right)\right) .
$$

Note that $d\left(y, A_{1}\right) \leq r_{1} q^{\text {lev } A_{1}}$ and observe

$$
\begin{aligned}
d(z, y) & \leq K_{X}\left(d\left(z, m_{A_{2}}\right)+d\left(m_{A_{2}}, y\right)\right) \\
& \leq 2 K_{X}^{2}\left(C_{2}+r_{2}\right) q^{\operatorname{lev} A_{2}},
\end{aligned}
$$


where we used Lemma 2.2 for the latter estimate. Combining our estimates yields

$$
d\left(z, A_{1}\right) \leq K_{X}\left(2 K_{X}^{2}\left(C_{2}+r_{2}\right) q^{\operatorname{lev} A_{2}-\operatorname{lev} A_{1}}+r_{1}\right) q^{\operatorname{lev} A_{1}},
$$

thus the assertion of the lemma follows.

\section{ADAPTIVE DYADIC GRIDS}

In this section we provide a customizable way to adapt dyadic grids, which is then applied in Section 4 to estimate the rearrangement operators $T_{m}$.

We recall that $K_{X}, C_{d}$ are constants defined by the quasimetric and the space of homogeneous type $X$ and $C_{1}, C_{2}$ are determined by the collection of dyadic cubes, cf. Section 2, For a given collection $\mathscr{A}$ of dyadic cubes in $X$ and $\alpha>0$ we define

$$
\mathscr{A}^{(\alpha)}:=\bigcup_{A \in \mathscr{A}}\left\{Q \in \mathscr{Q}_{\operatorname{lev} A}: Q \cap \alpha \diamond A \neq \emptyset\right\} .
$$

The following result is a version of the well known one-third-trick in spaces of homogeneous type.

Theorem 3.1. Let $C_{R}>0$ and $\mu \in \mathbb{N}$ be such that

$$
4 K_{X}^{3}\left(1+C_{2} / C_{R}\right) \cdot q^{\mu} \leq 1 .
$$

Let $\mathscr{A} \subset \mathscr{Q}$ be a finite collection of cubes satisfying

(1) the separation condition

$$
\left(C_{R} \diamond A_{1}\right) \cap\left(C_{R} \diamond A_{2}\right)=\emptyset
$$

for all $A_{1} \neq A_{2}$ in $\mathscr{A}$ with lev $A_{1}=\operatorname{lev} A_{2}$,

(2) the small successor condition

$$
\operatorname{lev} A \geq \mu+\operatorname{lev} \pi(A), \quad A \in \mathscr{A}^{(\alpha)},
$$

where $\alpha=2 K_{X}^{3}\left(C_{2}+C_{R}\right)+C_{R} / 2$ and $\pi \equiv \pi_{\mathscr{A}(\alpha)}$.

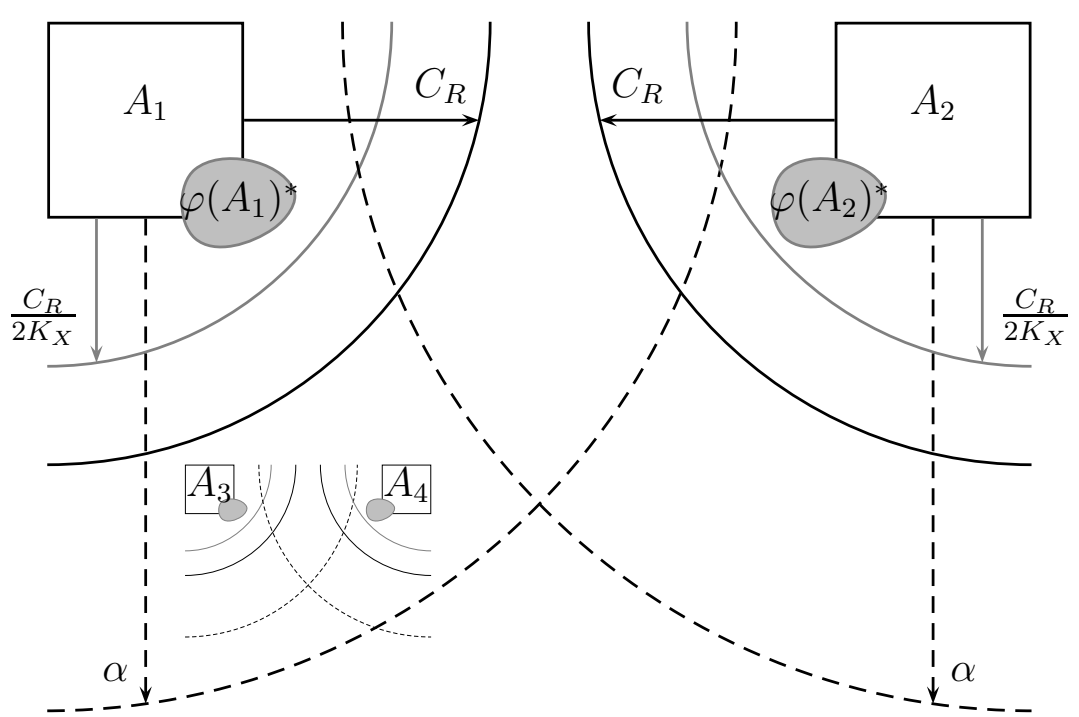

FiguRe 1. 
Let $\varphi: \mathscr{A} \rightarrow \mathscr{P}(\mathscr{A})$ be a map such that

$$
\begin{array}{lll}
\operatorname{lev} Q>\operatorname{lev} A, & A \in \mathscr{A} \text { and } Q \in \varphi(A), \\
\varphi(A)^{*} \subset \frac{C_{R}}{2 K_{X}} \diamond A, & A \in \mathscr{A} .
\end{array}
$$

Then there exist a collection $\mathscr{B}$ of adapted cubes in $X$ and a bijective map $\sigma: \mathscr{A} \rightarrow \mathscr{B}$ satisfying

$$
A \cup \sigma(\varphi(A))^{*} \subset \sigma(A) \subset C_{R} \diamond A, \quad A \in \mathscr{A} .
$$

and the measure estimate

$$
|\sigma(A)| \leq C_{d}\left(\frac{K_{X}\left(C_{2}+C_{R}\right)}{C_{1}}\right)^{\log _{2}\left(C_{d}\right)} \cdot|A|, \quad A \in \mathscr{A} .
$$

Moreover, the collection

is nested.

$$
\mathscr{B}=\{\sigma(A): A \in \mathscr{A}\}
$$

The hypotheses of Theorem 3.1 are visualized in Figure 1 .

Proof. We set $\widetilde{\mathscr{A}}_{j}:=\mathscr{A} \cap \mathscr{Q}_{j}, j \in \mathbb{Z}$. Let the sequence $j_{\ell}$ be such that $\widetilde{\mathscr{A}}_{j_{\ell}} \neq \emptyset$ and $\widetilde{\mathscr{A}}_{k}=\emptyset$ for all $j_{\ell-1}<k<j_{\ell}, \ell \leq 0$. Then define $\mathscr{A}_{\ell}:=\widetilde{\mathscr{A}}_{j_{\ell}}, \ell \leq 0$ and assume without restriction that $\mathscr{A}_{0}$ consists of the cubes in $\mathscr{A}$ with maximal level. The proof proceeds by induction on lev $A$ for cubes $A \in \mathscr{A}$, starting with cubes in $\mathscr{A}_{0}$.

Step 1. We begin the induction by defining

$$
\sigma(A):=A \text { for } A \in \mathscr{A}_{0} \quad \text { and } \quad \mathscr{B}_{0}:=\left\{\sigma(A): A \in \mathscr{A}_{0}\right\} .
$$

Observe that (3.7) holds for all $A \in \mathscr{A}_{0}$. Now, let $k<0$ and assume that all the cubes $\sigma(A), A \in \mathscr{A}_{j}$, and the collections $\mathscr{B}_{j}:=\left\{\sigma(A): A \in \mathscr{A}_{j}\right\}$ are already defined for all $j>k$. In order to construct $\sigma(A)$, let $A \in \mathscr{A}_{k}$ and define

$$
\sigma(A):=\left(A \cup \sigma(\varphi(A))^{*}\right) \cup\left(\bigcup\left\{B \in \mathscr{B}_{j}: j>k, B \cap\left(A \cup \sigma(\varphi(A))^{*}\right) \neq \emptyset\right\}\right) .
$$

We collect all those cubes in

$$
\mathscr{B}_{k}:=\left\{\sigma(A): A \in \mathscr{A}_{k}\right\} .
$$

Finally, the set $\mathscr{B}$ of all adapted cubes is defined as

$$
\mathscr{B}:=\bigcup_{j} \mathscr{B}_{j} .
$$

In the next two steps we will inductively verify the nestedness of $\mathscr{B}$ and the localization property (3.7).

Step 2. Here we prove the nestedness of $\mathscr{B}$. To this end, define the level of an adapted cube $B=\sigma(A)$ by lev $B=\operatorname{lev} A$. Let $B_{1}, B_{2} \in \mathscr{B}$ be such that $B_{1} \cap B_{2} \neq \emptyset$ and assume lev $B_{1} \leq \operatorname{lev} B_{2}$. If lev $B_{1}=\operatorname{lev} B_{2}$, then properties (3.3) and (3.7) yield $B_{1}=B_{2}$. So we may now assume that lev $B_{1}<\operatorname{lev} B_{2}$. Choose $A_{1} \in \mathscr{A}$ such that $\sigma\left(A_{1}\right)=B_{1}$. If $B_{2} \cap\left(A_{1} \cup \sigma\left(\varphi\left(A_{1}\right)\right)^{*}\right)=\emptyset$ we get $B_{1} \cap B_{2}=\emptyset$ by definition of $B_{1}$, cf. (3.10). This contradicts the assumption $B_{1} \cap B_{2} \neq \emptyset$. Thus, $B_{2} \cap\left(A_{1} \cup \sigma(\varphi(A))^{*}\right) \neq \emptyset$ and, by (3.10) again, we infer $B_{2} \subset B_{1}$, proving the nestedness of $\mathscr{B}$.

Step 3. In this step we will verify (3.7). Assume that (3.7) is true for all $A \in \mathscr{A}_{j}, j>k$. Recall that $\mathscr{B}$ is nested by Step 2 of this proof. Now, let $A \in \mathscr{A}_{k}$ be fixed. First, note 
that $A \cup \sigma(\varphi(A))^{*} \subset \sigma(A)$ by the definition of $\sigma(A)$, cf. (3.10). Secondly, we show that $\sigma(A) \subset C_{R} \diamond A$. Let $B \in \mathscr{B}_{j}, j>k$ be such that $B \cap\left(A \cup \sigma(\varphi(A))^{*}\right) \neq \emptyset$. The condition $B \cap\left(A \cup \sigma(\varphi(A))^{*}\right) \neq \emptyset$ is covered by the cases

(1) $B \cap \frac{C_{R}}{2 K_{X}} \diamond A \neq \emptyset$,

(2) there exists a $Q \in \varphi(A)$ such that $B \cap \sigma(Q) \neq \emptyset$, and so by (3.9) either

(a) $\sigma(Q) \subset B$ or

(b) $B \subset \sigma(Q)$.

First, let us consider case (1). Due to the induction hypothesis, (3.7) is true for $\sigma^{-1}(B)$, that is $B \subset C_{R} \diamond \sigma^{-1}(B)$. Thus, Lemma 2.3 implies

$$
B \subset C_{R} \diamond \sigma^{-1}(B) \subset r \diamond A,
$$

where $r=2 K_{X}^{3}\left(C_{2}+C_{R}\right) \cdot q^{\operatorname{lev} \sigma^{-1}(B)-\operatorname{lev} A}+C_{R} / 2$. Observe that since $r \leq \alpha$, we can find a cube $\widetilde{A} \in \mathscr{A}_{k}^{(\alpha)}$ such that $\sigma^{-1}(B) \subsetneq \widetilde{A}$. Hence lev $\sigma^{-1}(B) \geq \mu+\operatorname{lev} \widetilde{A}=\mu+\operatorname{lev} A$ and so $r \leq 2 K_{X}^{3}\left(C_{2}+C_{R}\right) \cdot q^{\mu}+\frac{C_{R}}{2}$. Since $r \leq C_{R}$ by (3.2), the inclusion $B \subset C_{R} \diamond A$ follows.

In case (2a), the first inclusion in (3.7) yields $Q \subset \sigma(Q) \subset B$. Since $Q \subset \varphi(A)^{*} \subset$ $\frac{C_{R}}{2 K_{X}} \diamond A$ by (3.6), in particular $B \cap \frac{C_{R}}{2 K_{X}} \diamond A \neq \emptyset$. Hence, case (2a) is covered by case (11). In case (2b), condition (3.6) implies $\sigma(Q) \cap \frac{C_{R}}{2 K_{X}} \diamond A \neq \emptyset$. Applying the proof of case (1) to $\sigma(Q)$ instead of $B$, we obtain $\sigma(Q) \subset C_{R} \diamond A$, and thus $B \subset C_{R} \diamond A$.

To summarize, in any of the cases (11), (2a) and (2b), the condition $B \cap\left(A \cup \sigma(\varphi(A))^{*}\right) \neq$ $\emptyset$ yields $B \subset C_{R} \diamond A$, which proves (3.7), i.e., $\sigma(A) \subset C_{R} \diamond A$.

Finally, the measure estimate (3.8) is an immediate consequence of the doubling condition (2.1) and

$$
B\left(m_{A}, C_{1} q^{\operatorname{lev} A}\right) \subset A \subset \sigma(A) \subset C_{R} \diamond A \subset B\left(m_{A}, K_{X}\left(C_{2}+C_{R}\right) q^{\operatorname{lev} A}\right),
$$

where the latter inclusion follows from Lemma 2.2 .

\section{REARRANGEMENT OPERATORS}

Following [13], we define and analyze rearrangement operators on spaces of homogeneous type, thereby extending the rearrangement operators $T_{m}$ introduced in [5], that act on the standard Haar system.

The shift relation $\tau$. Let $m \in \mathbb{R}, m>0$ and $\tau \subset \bigcup_{j \in \mathbb{Z}} \mathscr{Q}_{j} \times \mathscr{Q}_{j}$ have the properties

(P1) $Q \subset m \diamond P$ for all $(P, Q) \in \tau$, cf. Figure 2,

(P2) there exists a finite partition $\tau_{1}, \ldots, \tau_{M}$ of $\tau$ such that $\tau_{k}$ is a bijective function for all $1 \leq k \leq M$.

The relation $\tau$ generalizes the classical shift $I \mapsto I+m|I|$ on $\mathbb{R}$, cf. [5].

In order to apply Theorem 3.1 to our shift $\tau$, we decompose $\tau_{k}$ into suitable subcollections in the following way.

(C1) First, let us choose a constant $C_{R}>0$ and split $\tau_{k}$ into the collections $\mathscr{G}_{k, 1}, \ldots, \mathscr{G}_{k, M_{k}}$, for all $1 \leq k \leq M$ such that

$$
\left(C_{R} \diamond \tau_{k}^{i}\left(A_{1}\right)\right) \cap\left(C_{R} \diamond \tau_{k}^{n}\left(A_{2}\right)\right)=\emptyset
$$

for all $A_{1}, A_{2} \in \operatorname{pr}_{1}\left(\mathscr{G}_{k, j}\right), 1 \leq j \leq M_{k}, i, n \in\{0,1\}$, where $\tau_{k}^{i}(A)$ is defined to be $A$ for $i=0$ and $\tau_{k}(A)$ for $i=1$. The projection onto the first and second coordinates of a relation are denoted by $\mathrm{pr}_{1}$ and $\mathrm{pr}_{2}$, respectively. Observe that the constants $M_{k}, 1 \leq k \leq M$ depend only on $X$, cf. [13]. We refer to Figure 2 for a picture of the separation condition (4.1). 


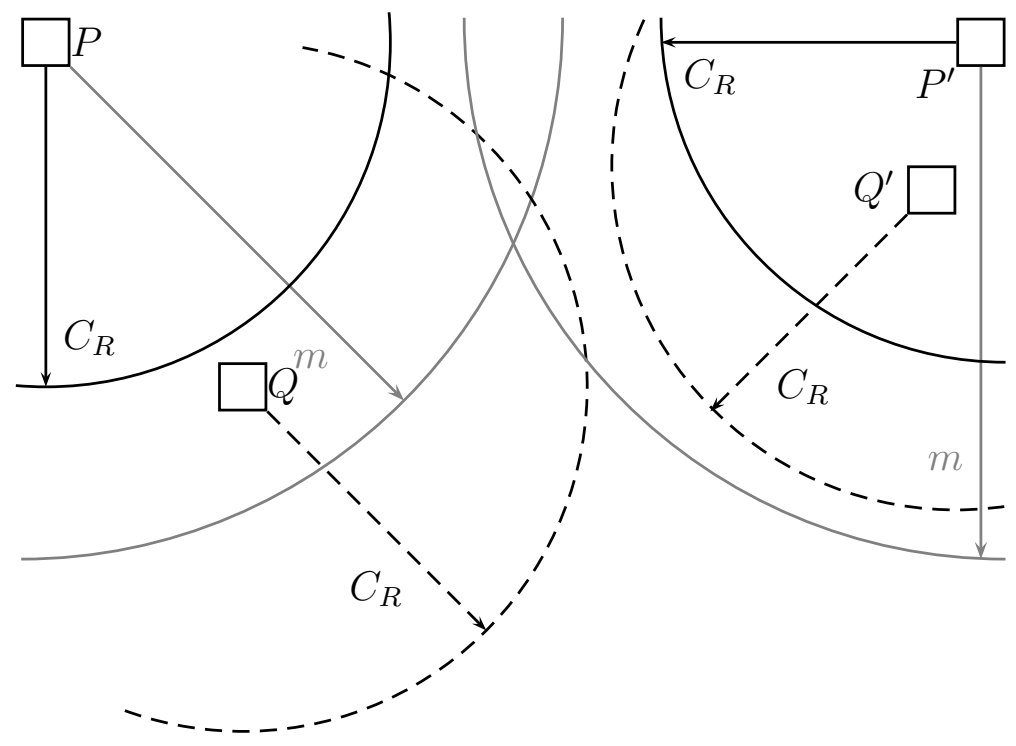

FIGURE 2.

(C2) Secondly, let $\ell$ be a positive integer and define

$$
\mathscr{H}_{k, j, i}^{(\ell)}=\mathscr{G}_{k, j} \cap\left(\bigcup_{r \in \mathbb{Z}} \mathscr{Q}_{i+r \ell} \times \mathscr{Q}_{i+r \ell}\right),
$$

for all $1 \leq k \leq M, 1 \leq j \leq M_{k}, 0 \leq i \leq \ell-1$. The parameter $\ell$ will later be chosen to be approximately $\log (2+m)$ with $m$ being the parameter from (P1).

(C3) Finally, define

$$
\psi_{k, j, i}^{(\ell)}(A)=\left\{(P, Q) \in \mathscr{H}_{k, j, i}^{(\ell)}: P \subsetneq A \text { or } Q \subsetneq A\right\},
$$

for all $A \in\left(\operatorname{pr}_{1}\left(\mathscr{H}_{k, j, i}^{(\ell)}\right) \cup \operatorname{pr}_{2}\left(\mathscr{H}_{k, j, i}^{(\ell)}\right)\right)$.

The collections $\psi_{k, j, i}^{(\ell)}(A)$ are well localized around $A$, which is discussed in

Lemma 4.1. Let $m \in \mathbb{R}, m>0$ and let $\ell$ be a positive integer. Then

$$
\left(\operatorname{pr}_{1}\left(\psi_{k, j, i}^{(\ell)}(A)\right) \cup \operatorname{pr}_{2}\left(\psi_{k, j, i}^{(\ell)}(A)\right)\right)^{*} \subset\left(c_{1}(1+m) q^{\ell}\right) \diamond A,
$$

for all $A \in \mathscr{H}_{k, j, i}^{(\ell)}, 1 \leq k \leq M, 1 \leq j \leq M_{k}, 0 \leq i \leq \ell-1$. The constant $c_{1}$ depends only on the space of homogeneous type $X$.

Proof. Let $(P, Q) \in \psi_{k, j, i}^{(\ell)}(A)$. Then, $P \subsetneq A$ or $Q \subsetneq A$ by definition of $\psi_{k, j, i}^{(\ell)}$. We know from (P1) that $P \cup Q \subset m \diamond P$, hence Lemma 2.3 yields

$$
P \cup Q \subset\left(2 K_{X}^{3}\left(C_{2}+m\right) q^{\operatorname{lev} P-\operatorname{lev} A} \diamond A\right) .
$$

Noting that lev $P \geq \ell+\operatorname{lev} A$ by (C2 $)$ concludes the proof.

The Shift operator $T$. In order to define analogues of $T_{m}$ on spaces of homogeneous type, we need a substitute $\left\{h_{Q}\right\}$ for the standard Haar system. We require the system of functions $\left\{h_{Q}\right\}_{Q \in \mathscr{Q}}$ to satisfy the conditions

(H1) $\operatorname{supp} h_{Q} \subset Q$, for all $Q \in \mathscr{Q}$,

(H2) $\left\|h_{Q}\right\|_{\infty} \leq C_{h} \frac{1}{|P|+|Q|} \int\left|h_{P}\right|$, for all $(P, Q) \in \tau$,

(H3) for each $k$ the collections $\left\{h_{P}: P \in \operatorname{pr}_{1}\left(\tau_{k}\right)\right\}$ and $\left\{h_{Q}: Q \in \operatorname{pr}_{2}\left(\tau_{k}\right)\right\}$ constitute martingale difference sequences, separately. 
The constant $C_{h}>0$ is independent of $(P, Q)$. The collections $\mathscr{H}_{k, j, i}^{(\ell)}$, defined in (C2), naturally induce the subspaces $H_{k, j, i}^{(\ell)}$ of $L_{E}^{p}(X)$ given by

$$
H_{k, j, i}^{(\ell)}=\left\{f \in L_{E}^{p}(X): f=\sum_{P \in \operatorname{pr}_{1}\left(\mathscr{H}_{k, j, i}^{(\ell)}\right)}\left\langle f, h_{P}\right\rangle h_{P}\right\} .
$$

We now define the shift operators $T_{k}$ induced by $\tau_{k}, 1 \leq k \leq M$, as the linear extension of the map

$$
h_{P} \mapsto \begin{cases}h_{Q}, & \text { if }(P, Q) \in \tau_{k}, \\ 0, & \text { otherwise. }\end{cases}
$$

If the collections $\psi_{k, j, i}^{(\ell)}$ are sufficiently localized, then the operators $T_{k}$ are bounded on the subspace $H_{k, j, i}^{(\ell)}$. The details are given in the theorem below.

Theorem 4.2. Let $X$ be a space of homogeneous type, $E$ a UMD-space and $1<p<\infty$. Let $m \in \mathbb{R}, m>0$, then there exists a constant $\beta>0$ such that for all integers $\ell$ satisfying

$$
(1+m) q^{\ell} \leq \beta
$$

we have

$$
\left\|T_{k} f\right\|_{L_{E}^{p}(X)} \leq C\|f\|_{L_{E}^{p}(X)}, \quad f \in H_{k, j, i}^{(\ell)}
$$

for all $1 \leq k \leq M, 1 \leq j \leq M_{k}, 0 \leq i \leq \ell-1$. The constant $C$ depends only on $p, X$ and $E$, and the constant $\beta$ only on $X$.

Proof. Let $\ell$ be fixed throughout the proof satisfying (4.3). Conditions on the constant $\beta$ will be imposed within the proof.

Our goal is to apply Theorem 3.1 to each of the collections $\mathscr{H}_{k, j, i}^{(\ell)}$. With $k, j, i$ fixed, let us define the collections $\mathscr{C}=\mathscr{C}^{(1)} \cup \mathscr{C}^{(2)}=\operatorname{pr}_{1}\left(\mathscr{H}_{k, j, i}^{(\ell)}\right) \cup \operatorname{pr}_{2}\left(\mathscr{H}_{k, j, i}^{(\ell)}\right)$ and let

$$
\mathscr{A} \subset \mathscr{C} \text { be a finite set. }
$$

The function $\varphi: \mathscr{A} \rightarrow \mathscr{P}(\mathscr{A})$ is given by

$$
\varphi(A):=\operatorname{pr}_{1}\left(\psi_{k, j, i}^{(\ell)}(A)\right) \cup \operatorname{pr}_{2}\left(\psi_{k, j, i}^{(\ell)}(A)\right), \quad A \in \mathscr{A},
$$

where $\psi_{k, j, i}^{(\ell)}$ is defined in (C3). We shall now verify that $\mathscr{A}$ and $\varphi$ satisfy the hypotheses of Theorem 3.1 .

First, observe that the separation condition (3.3) is satisfied due to (C1). Secondly, let $\mu=\ell$, then (3.2) holds for sufficiently small $\beta$, where the constraint for $\beta$ depends only on $X$. Additionally, observe that (C2) implies (3.4). From Lemma 4.1 and (4.3) it follows that $\varphi(A)^{*} \subset \frac{C_{R}}{2 K_{X}} \diamond A$ if $\beta$ is sufficiently small. Having verified all the hypotheses of Theorem 3.1 , we obtain a nested collection of sets $\mathscr{B}$ and a bijective map $\sigma: \mathscr{A} \rightarrow \mathscr{B}$, such that

$$
A \cup \sigma(\varphi(A))^{*} \subset \sigma(A) \subset C_{R} \diamond A \quad \text { and } \quad|\sigma(A)| \leq c_{2}\left(1+C_{R}\right)^{\log _{2}\left(C_{d}\right)} \cdot|A|
$$

for all $A \in \mathscr{A}$. The constant $c_{2}$ depends only on $X$.

Let us now define a nested collection of sets supporting the shifts $\tau$ inductively, beginning with the smallest cubes. Set $n_{\max }=\max \{\operatorname{lev}(A): A \in \mathscr{A}\}$ and define

$$
\theta(P):=\theta(Q):=\sigma(P) \cup \sigma(Q)
$$

for all $(P, Q) \in \mathscr{H}_{k, j, i}^{(\ell)}$ such that $\operatorname{lev}(P)=n_{\max }$. With $n<n_{\max }$ fixed, assume that $\theta(A)$ is already defined for all cubes $A$ satisfying $\operatorname{lev}(A)>n$. The function $\theta$ is specified by

$$
\theta(P):=\theta(Q):=\sigma(P) \cup \sigma(Q) \cup\left\{\theta(R): \operatorname{lev}_{8} R>\operatorname{lev} P, \theta(R) \cap(\sigma(P) \cup \sigma(Q)) \neq \emptyset\right\}^{*},
$$


for all $(P, Q) \in \mathscr{H}_{k, j, i}^{(\ell)}$ with $\operatorname{lev}(P)=n$. As an immediate consequence of the principle of construction, the collection $\{\theta(A): A \in \mathscr{A}\}$ is nested and

$$
P \cup Q \subset \theta(P)=\theta(Q), \quad(P, Q) \in \mathscr{H}_{k, j, i}^{(\ell)}, P \in \mathscr{A} .
$$

Furthermore, a straightforward calculation using Lemma 2.3 and (4.3) shows that there exists a constant $c_{3}$ depending only on $X$ such that

$$
\theta(P) \subset\left(c_{3} \diamond P\right) \cup\left(c_{3} \diamond Q\right), \quad(P, Q) \in \mathscr{H}_{k, j, i}^{(\ell)}, P \in \mathscr{A},
$$

if $\beta$ is sufficiently small. From the latter inclusion we obtain

$$
\theta(P) \leq c_{4}(|P|+|Q|), \quad(P, Q) \in \mathscr{H}_{k, j, i}^{(\ell)}, P \in \mathscr{A},
$$

where $c_{4}$ depends only on $X$. Let us define the filtration $\left\{\mathcal{F}_{n}\right\}$ by

$$
\mathcal{F}_{n}=\sigma \text {-algebra }(\{\theta(A): A \in \mathscr{A}, \text { lev } A \leq n\}), \quad n \in \mathbb{Z} .
$$

Observe that $\theta(A)$ is an atom in $\mathcal{F}_{\text {lev } A}$ for all $A \in \mathscr{A}$, since $\{\theta(A): A \in \mathscr{A}\}$ is a nested collection. Thus, (H2) and (4.5) imply

$$
\left|h_{\tau_{k}(A)}\right| \leq c_{5} \mathbb{E}\left(\left|h_{A}\right| \mid \mathcal{F}_{n}\right), \quad A \in \mathscr{E}_{n},
$$

where $\mathscr{E}_{n}=\mathscr{Q}_{n} \cap \mathscr{A} \cap \mathscr{C}^{(1)}$ and $c_{5}$ depends only on $X$ and $C_{h}$.

We will now estimate $T f$ for all $f \in H_{k, j, i}^{(\ell)}$. Note that (H3) and the UMD-property of $E$ allow us to assume that $f$ is of the form

$$
f=\sum_{n} \sum_{A \in \mathscr{E}_{n}}\left\langle f, h_{A}\right\rangle h_{A} .
$$

Moreover, $\left.T\right|_{H_{k, j, i}^{(\ell)}}=\left.T_{k}\right|_{H_{k, j, i}^{(\ell)}}$ is a function due to (P2). By employing (H[3) again, we introduce Rademacher means in $\left\|T_{k} f\right\|$ and obtain

$$
\begin{aligned}
\left\|T_{k} f\right\| & =\left\|\sum_{n} \sum_{A \in \mathscr{E}_{n}}\left\langle f, h_{A}\right\rangle h_{\tau_{k}(A)}\right\| \\
& \approx \int_{0}^{1}\left\|\sum_{n} r_{n}(t) \sum_{A \in \mathscr{E}_{n}}\left\langle f, h_{A}\right\rangle h_{\tau_{k}(A)}\right\| \mathrm{d} t .
\end{aligned}
$$

Furthermore, estimate (4.6) yields

$$
\begin{aligned}
\left\|T_{k} f\right\| & \approx \int_{0}^{1}\left\|\sum_{n} r_{n}(t) \sum_{A \in \mathscr{E}_{n}}\left\langle f, h_{A}\right\rangle\left|h_{\tau_{k}(A)}\right|\right\| \mathrm{d} t \\
& \lesssim \int_{0}^{1}\left\|\sum_{n} r_{n}(t) \mathbb{E}\left(\sum_{A \in \mathscr{E}_{n}}\left\langle f, h_{A}\right\rangle\left|h_{A}\right| \mid \mathcal{F}_{n}\right)\right\| \mathrm{d} t
\end{aligned}
$$

by means of Kahane's contraction principle. Applying Bourgain's version of Stein's martingale inequality gives us

$$
\left\|T_{k} f\right\| \lesssim \int_{0}^{1}\left\|\sum_{n} r_{n}(t) \sum_{A \in \mathscr{E}_{n}}\left\langle f, h_{A}\right\rangle\left|h_{A}\right|\right\| \mathrm{d} t .
$$

Using Kahane's contraction principle and the UMD-property, cf. (H[3]), concludes the proof. 
Combining the estimates of Theorem 4.2 on the subspaces $H_{k, j, i}^{(\ell)}$, we obtain estimates for $T_{k}$ on $\overline{\operatorname{span}}\left\{h_{P}: P \in \mathscr{Q}\right\}$ in the subsequent theorem, cf. [5].

Theorem 4.3. Let $X$ be a space of homogeneous type, $E$ a UMD-space, $1<p<\infty$ and $m \in \mathbb{R}, m>0$. Then for all $1 \leq k \leq M$ the linear operator $T_{k}$ satisfies

$$
\left\|T_{k} f\right\|_{L_{E}^{p}(X)} \leq C \log (2+m)^{\alpha}\|f\|_{L_{E}^{p}(X)}, \quad f \in \overline{\operatorname{span}}\left\{h_{P}: P \in \mathscr{Q}\right\} .
$$

If $L_{E}^{p}(X)$ has type $\mathcal{T}$ and cotype $\mathcal{C}$, then $\alpha<1$ is given by $1 / \mathcal{T}-1 / \mathcal{C}$. The constant $C$ depends only on $p, X, E$ and $\alpha$.

Proof. Within this proof we shall abbreviate $\|\cdot\|_{L_{E}^{p}(X)}$ by $\|\cdot\|$. Let $m>0$ and choose $\ell$ as the minimal integer satisfying (4.3), i.e., there exists a constant $c_{1}$ only depending on $X$ with

$$
\ell \geq c_{1} \log (2+m)
$$

Assume that $f$ is a finite sum of the form $f=\sum_{j=1}^{M_{k}} \sum_{i=0}^{\ell-1} \sum_{P \in \mathscr{H}_{k, j, i}^{(\ell)}} f_{P} h_{P}$. Then, by definition of $T_{k}$ and the UMD-property of $L_{E}^{p}(X)$ applied to (H3), we obtain

$$
\left\|T_{k} f\right\| \lesssim \int_{0}^{1}\left\|\sum_{j, i} r_{j, i}(t) T_{k} d_{j, i}\right\| \mathrm{d} t
$$

where $d_{j, i}=\sum_{P \in \mathscr{H}_{k, j, i}^{(\ell)}} f_{P} h_{P}$. The type inequality yields

$$
\left\|T_{k} f\right\| \lesssim\left(\sum_{j, i}\left\|T_{k} d_{j, i}\right\|^{\mathcal{T}}\right)^{1 / \mathcal{T}}
$$

where $L_{E}^{p}(X)$ is of type $\mathcal{T}$. Theorem 4.2 implies $\left\|T_{k} d_{j, i}\right\| \lesssim\left\|d_{j, i}\right\|$, hence

$$
\left\|T_{k} f\right\| \lesssim\left(M_{k} \cdot \ell\right)^{1 / \mathcal{T}-1 / \mathcal{e}}\left(\sum_{j, i}\left\|d_{j, i}\right\|^{\mathfrak{e}}\right)^{1 / \mathcal{e}}
$$

where $L_{E}^{p}(X)$ is of cotype $\mathcal{C}$. The cotype inequality and the UMD-property show

$$
\left\|T_{k} f\right\| \lesssim\left(M_{k} \cdot \ell\right)^{1 / \mathcal{T}-1 / \mathcal{C}}\|f\| .
$$

Since $M_{k}$ depends only on $X$, using (4.8) gives (4.7) for finite sums $f$ in $\operatorname{span}\left\{h_{P}: P \in \mathscr{Q}\right\}$, thus concluding the proof by unique extension.

\section{STRIPE OPERATOR}

In this section we define stripe operators on spaces of homogeneous type and provide vector-valued $L^{p}$ estimates. Our notion of stripe operators generalizes those on $\mathbb{R}^{k}$ analyzed in [9], which will now be briefly reviewed.

For a positive integer $\lambda$, the stripes $\mathscr{S}_{\lambda}^{(m)}$ of the dyadic cube $[0,1]^{n}$ are given by

$$
\mathscr{S}_{\lambda}^{(m)}\left([0,1]^{k}\right)=\left\{\begin{array}{l}
Q: \begin{array}{l}
Q \text { is a dyadic cube with }|Q|=2^{-\lambda k} \\
Q \subset\left[\frac{m-1}{2^{\lambda}}, \frac{m}{2^{\lambda}}\right] \times[0,1]^{k-1}
\end{array}
\end{array}\right\}
$$

where $1 \leq m \leq 2^{\lambda}$. For an arbitrary dyadic cube $A$, the stripes $\mathscr{S}_{\lambda}^{(m)}(A)$ are obtained by scaling and translating $\mathscr{S}_{\lambda}^{(m)}\left([0,1]^{k}\right)$ to the position of $A$ in the dyadic grid. The stripe 
operators $S_{\lambda}^{(m)}$ are defined by

$$
S_{\lambda}^{(m)} h_{A}:=g_{A, \lambda}^{(m)}:=\sum_{R \in \mathscr{S}_{\lambda}^{(m)}(A)} h_{R},
$$

where $h_{A}$ and $h_{R}$ denote canonical Haar functions supported on the dyadic cubes $A$ and $R$. Estimates for $S_{\lambda}^{(m)}$ on $L^{p}$ were used in [11] as well as in [9] to show weak lower semicontinuity for functionals with separately convex integrands on scalar and vector-valued $L^{p}$, respectively.

We will now extend the operators $S_{\lambda}^{(m)}$ and their vector-valued estimates to spaces of homogeneous type.

The stripes $\mathscr{S}_{\lambda}^{(m)}$. Let $\lambda$ and $M$ be positive integers and define the stripes $\mathscr{S}_{\lambda}^{(m)}(A)$, $A \in \mathscr{Q}, 1 \leq m \leq M$ as arbitrary subsets of $\{B \subset A$ : lev $B=\operatorname{lev} A+\lambda\}$ satisfying the conditions

(S1) $A=\bigcup_{m=1}^{M} \mathscr{S}_{\lambda}^{(m)}(A)^{*}$ is a disjoint union,

(S2) there exists an absolute constant $K_{1}$ such that

$$
\left|\mathscr{S}_{\lambda}^{(m)}(A)^{*}\right| \leq K_{1}\left|\mathscr{S}_{\lambda}^{(n)}(A)^{*}\right|, \quad 1 \leq m, n \leq M,
$$

(S3) $\left\{\mathscr{S}_{\lambda}^{(m)}(A)^{*}: A \in \mathscr{Q}\right\}$ is nested, with $1 \leq m \leq M$ being fixed,

(S4) there exist constants $\varepsilon>0$ and $K_{2}$ depending only on $X$ such that for all $1 \leq$ $m \leq M$ we have

$$
\left|\mathscr{E}_{j}^{(m)}(A)^{*}\right| \leq K_{2} q^{j \varepsilon}|A|, \quad 0 \leq j \leq \lambda-1,
$$

where

$$
\mathscr{E}_{j}^{(m)}(A):=\left\{B \in \mathscr{Q}_{\operatorname{lev} A+j}: B \cap \mathscr{S}_{\lambda}^{(m)}(A)^{*} \neq \emptyset\right\}
$$

The classical stripe (5.1) defined in $\mathbb{R}^{k}$ equipped with the Euclidean metric satisfies the conditions (S1) to (S4) with parameters $M=2^{\lambda}, K_{1}=1, K_{2}=1, q=1 / 2$ and $\varepsilon=1$.

The stripe operators $S_{\lambda}^{(m)}$. Let the collection of functions $\left\{h_{A}: A \in \mathscr{Q}\right\}$ suffice

(M1) $\operatorname{supp} h_{A} \subset A$, for all $A \in \mathscr{Q}$,

(M2) $\left\{h_{A}: A \in \mathscr{Q}\right\}$ constitutes a martingale difference sequence.

Moreover, let $\left\{g_{A, \lambda}^{(m)}: A \in \mathscr{Q}\right\}, 1 \leq m \leq M$ be collections of functions that satisfy

(G1) $\operatorname{supp} g_{A, \lambda}^{(m)} \subset \mathscr{S}_{\lambda}^{(m)}(A)$, for all $A \in \mathscr{Q}$ and $1 \leq m \leq M$,

(G2) $\left\{g_{A, \lambda}^{(m)}: 1 \leq m \leq M, A \in \mathscr{Q}\right\}$ constitutes a martingale difference sequence,

(G3) $\left\|g_{A, \lambda}^{(m)}\right\|_{\infty} \leq C_{g} \frac{1}{\left|\mathscr{S}_{\lambda}^{(n)}(A)^{*}\right|} \int\left|g_{A, \lambda}^{(n)}\right|$, for all $A \in \mathscr{Q}, 1 \leq m, n \leq M, m \neq n$ and some constant $C_{g} \geq 1$.

We define the stripe operator $S_{\lambda}^{(m)}, 1 \leq m \leq M$, as the linear extension of

$$
S_{\lambda}^{(m)} h_{A}:=g_{A, \lambda}^{(m)}, \quad A \in \mathscr{Q} .
$$

Note that the classical stripe operator (5.2) satisfies all of the above conditions.

Lemma 5.1. Let $g_{A, \lambda}^{(m)}$ and $g_{A, \lambda}^{(n)}$ be stripe functions satisfying (G11) and (G[3), then

$$
\left|\left\{\left|g_{A, \lambda}^{(n)}\right| \geq \frac{\left\|g_{A, \lambda}^{(m)}\right\|_{\infty}}{2 C_{g}}\right\}\right| \geq \frac{1}{2 C_{g}^{2}}\left|\mathscr{S}_{\lambda}^{(n)}(A)^{*}\right| .
$$


Proof. We shall abbreviate $g^{(m)}=g_{A, \lambda}^{(m)}, g^{(n)}=g_{A, \lambda}^{(m)}$ and $\mathscr{S}=\mathscr{S}_{\lambda}^{(n)}(A)$. Assume the contrary, that is

$$
\left|\left\{\left|g^{(n)}\right| \geq \frac{\left\|g^{(m)}\right\|_{\infty}}{2 C_{g}}\right\}\right|<\frac{1}{2 C_{g}^{2}}\left|\mathscr{S}^{*}\right|
$$

Then, (G3) implies

$$
\begin{aligned}
\frac{\left|\mathscr{S}^{*}\right|}{C_{g}} \cdot\left\|g^{(m)}\right\|_{\infty} \leq \int_{\mathscr{S}^{*}}\left|g^{(n)}\right| \leq \mid\{ & \left.\left|g^{(n)}\right|<\frac{\left\|g^{(m)}\right\|_{\infty}}{2 C_{g}}\right\} \mid \cdot \frac{\left\|g^{(m)}\right\|_{\infty}}{2 C_{g}} \\
& +\left|\left\{\left|g^{(n)}\right| \geq \frac{\left\|g^{(m)}\right\|_{\infty}}{2 C_{g}}\right\}\right| \cdot\left\|g^{(n)}\right\|_{\infty} .
\end{aligned}
$$

Observe that (G[3) and (G1) give us $\left\|g^{(n)}\right\|_{\infty} \leq C_{g}\left\|g^{(m)}\right\|$, thus inserting (G1) and (5.4) in the latter display yields a contradiction, proving the lemma.

The subsequent results, i.e., the combinatorial Lemma 5.2 and the estimates on stripe operators Theorems 5.3 and 5.4 are proved by similar methods as their Euclidean counterparts in [9].

Lemma 5.2. Let $\lambda$ and $k$ be positive integers. Then there exists a constant $K_{3}$ depending only on $X$ such that

$$
\left|\mathscr{S}_{\lambda}^{(m)}(A)^{*} \cap\left(\bigcup_{B \in \mathscr{E}^{(m)}(A)} \mathscr{S}_{\lambda}^{(m)}(B)^{*} \cup \mathscr{S}_{\lambda}^{(n)}(B)^{*}\right)\right| \leq K_{3} q^{k \cdot \varepsilon} \cdot\left|\mathscr{S}_{\lambda}^{(m)}(A)^{*}\right|
$$

for all $1 \leq m, n \leq M$ and $A \in \mathscr{Q}$, where

$$
\mathscr{E}^{(m)}(A):=\bigcup\left\{\mathscr{E}_{d \cdot k}^{(m)}(A): d \in \mathbb{N}, 1 \leq d \cdot k \leq \lambda-1\right\}
$$

Proof. First, observe that

$$
\begin{aligned}
\left|\mathscr{S}_{\lambda}^{(m)}(A)^{*} \cap\left(\bigcup_{B \in \mathscr{E}^{(m)}(A)} \mathscr{S}_{\lambda}^{(m)}(B)^{*} \cup \mathscr{S}_{\lambda}^{(n)}(B)^{*}\right)\right| \\
\leq \sum_{B \in \mathscr{E}^{(m)}(A)}\left|\mathscr{S}_{\lambda}^{(m)}(B)^{*}\right|+\left|\mathscr{S}_{\lambda}^{(n)}(B)^{*}\right| .
\end{aligned}
$$

Now we use (\$2) to dominate this expression by

$$
\left(1+K_{1}\right) \sum_{B \in \mathscr{E}^{(m)}(A)}\left|\mathscr{S}_{\lambda}^{(m)}(B)^{*}\right| .
$$

Note that (S1) and (S2) also give us

$$
\left|\mathscr{S}_{\lambda}^{(m)}(B)^{*}\right| \leq \frac{K_{1}}{M}|B| .
$$

The latter inequality implies that (5.5) is bounded from above by

$$
\frac{\left(1+K_{1}\right) K_{1}}{M}\left(\sum_{d: 1 \leq d \cdot k<\lambda} \sum_{B \in \mathscr{E}_{d \cdot k}^{(m)}(A)}|B|\right) .
$$

Employing (\$4) we estimate (5.6) by

$$
\frac{\left(1+K_{1}\right) K_{1} K_{2}}{M}\left(\sum_{\substack{d: 1 \leq d \cdot k<\lambda \\ 12}} q^{d \cdot k \cdot \varepsilon}|A|\right) .
$$


Finally, applying (\$2) concludes the proof of the lemma.

Theorem 5.3. Let $X$ be a space of homogeneous type, $E$ a UMD-space and $1<p<\infty$. Let $\lambda$ be a positive integer, then there exists a constant $C$ such that

$$
\left\|S_{\lambda}^{(m)} f\right\|_{L_{E}^{p}(X)} \leq C\left\|S_{\lambda}^{(n)} f\right\|_{L_{E}^{p}(X)}, \quad f \in \overline{\operatorname{span}}\left\{h_{Q}: Q \in \mathscr{Q}\right\},
$$

for all $1 \leq m, n \leq M$. The constant $C$ depends only on $p, X$ and $E$.

Proof. Let $\lambda \geq 1$ and $m \neq n$ be fixed throughout the proof. Define $k$ as the smallest positive integer such that $K_{3} q^{k \cdot \varepsilon} \leq \frac{1}{4 C_{g}^{2}}$, where $K_{3}, \varepsilon$ and $C_{g}$ are the constants appearing in Lemma 5.2, (\$44) and (G[3), respectively. Moreover, define the collections

$$
\begin{array}{rlrl}
\mathscr{C}_{j, \nu}^{(\delta)} & :=\bigcup_{\substack{0 \leq i \leq \lambda-1 \\
i \bmod k=\nu}} \mathscr{Q}_{(2 j+\delta) \lambda+i}, & j \in \mathbb{Z}, \delta \in\{0,1\}, 0 \leq \nu \leq k-1, \\
\mathscr{C}_{\nu}^{(\delta)}:=\bigcup_{j \in \mathbb{Z}} \mathscr{C}_{j, \nu}^{(\delta)}, & \delta \in\{0,1\}, 0 \leq \nu \leq k-1 .
\end{array}
$$

With $\nu$ and $\delta$ fixed, set

$$
A(Q):=\left(\mathscr{S}_{\lambda}^{(m)}(Q)^{*} \cup \mathscr{S}_{\lambda}^{(n)}(Q)^{*}\right) \backslash \bigcup_{\substack{P \in \mathscr{C}_{j, \nu}^{(\delta)} \\ \operatorname{lev} P>\operatorname{lev} Q}} A(P), \quad Q \in \mathscr{C}_{j, \nu}^{(\delta)}
$$

for each $j \in \mathbb{Z}$. This definition is understood as an induction on lev $Q$, starting with the maximal level in $\mathscr{C}_{j, \nu}^{(\delta)}$. Note that the above union is empty if lev $Q$ is maximal in $\mathscr{C}_{j, \nu}^{(\delta)}$. Now, Lemma 5.2 and our choice of $k$ imply

$$
\left|A(Q) \cap \mathscr{S}_{\lambda}^{(n)}(Q)^{*}\right| \geq\left(1-\frac{1}{4 C_{g}^{2}}\right)\left|\mathscr{S}_{\lambda}^{(n)}(Q)^{*}\right| .
$$

We collect all the sets $A(Q)$ in $\mathscr{A}$, to be more precise

$$
\mathscr{A}:=\left\{A(Q): Q \in \mathscr{C}_{\nu}^{(\delta)}\right\} .
$$

The inductive construction of $A(Q)$ is performed in such a way that $\mathscr{A}$ is nested, which we shall show in the following. Observe, if $P, Q \in \mathscr{C}_{j, \nu}^{(\delta)}$, then $A(P) \cap A(Q)=\emptyset$. Moreover, if $Q \in \mathscr{C}_{j, \nu}^{(\delta)}$, then $A(Q)$ comprises of cubes in $\mathscr{Q}_{\operatorname{lev} Q+2 \lambda-1}$. Thus, if $P \in \mathscr{C}_{i, \nu}^{(\delta)}$ and $Q \in \mathscr{C}_{j, \nu}^{(\delta)}$ with $i<j$, then $A(Q) \subset Q \subset A(P)$, provided $A(P) \cap A(Q) \neq \emptyset$. Hence, $\mathscr{A}$ is a nested collection. Let us define

$$
\mathscr{A}_{j}:=\left\{A(Q) \in \mathscr{A}: Q \in \mathscr{Q}_{j}\right\}, \quad j \in \mathbb{Z},
$$

and the filtrations $\left\{\mathcal{F}_{j}\right\}$ and $\left\{\mathcal{G}_{j}\right\}$ by

$$
\mathcal{F}_{j}:=\sigma \text {-algebra }\left(\bigcup_{i \leq j} \mathscr{A}_{i}\right), \quad \mathcal{G}_{j}:=\sigma \text {-algebra }\left(\left\{\mathscr{S}_{\lambda}^{(m)}(Q)^{*}: Q \in \mathscr{Q}_{i}, i \leq j\right\}\right)
$$

for all $j \in \mathbb{Z}$. Note that some of the sets $\mathscr{A}_{j}$ are empty. So, if $\mathscr{A}_{j}=\emptyset$, we delete the $\sigma$-algebras $\mathcal{F}_{j}$ and $\mathcal{G}_{j}$ from their respective filtrations.

Let $f \in L_{E}^{p}(X)$ have the representation $f=\sum_{Q \in \mathscr{C}_{\nu}^{(\delta)}} f_{Q} h_{Q}$. Due to (G(2), the UMDproperty and Kahane's contraction principle yield

$$
\left\|S_{\lambda}^{(m)} f\right\|=\left\|\sum_{Q \in \mathscr{C}_{\nu}^{(\delta)}} f_{Q} g_{Q, \lambda}^{(m)}\right\| \lesssim \int_{0}^{1}\left\|\sum_{Q \in \mathscr{C}_{\nu}^{(\delta)}} r_{Q}(t) f_{Q}\left|g_{Q, \lambda}^{(m)}\right|\right\| \mathrm{d} t .
$$


First, observe that

$$
\mathbb{1}_{\mathscr{S}_{\lambda}^{(m)}(Q)^{*}} \leq \frac{\left|\mathscr{S}_{\lambda}^{(m)}(Q)^{*}\right|}{|A(Q)|} \mathbb{E}\left(\mathbb{1}_{A(Q)} \mid \mathcal{G}_{\operatorname{lev} Q}\right), \quad Q \in \mathscr{C}_{\nu}^{(\delta)} .
$$

Secondly, due to our choice of $k$, we obtain from Lemma 5.2 that $\left|\mathscr{S}_{\lambda}^{(m)}(Q)^{*}\right| \leq \frac{4}{3}|A(Q)|$ for all $Q \in \mathscr{C}_{\nu}^{(\delta)}$. The latter two inequalities imply

$$
\left|g_{Q, \lambda}^{(m)}\right| \leq\left\|g_{Q, \lambda}^{(m)}\right\|_{\infty} \cdot \mathbb{1}_{\mathscr{S}_{\lambda}^{(m)}(Q)^{*}} \leq \frac{4}{3}\left\|g_{Q, \lambda}^{(m)}\right\|_{\infty} \cdot \mathbb{E}\left(\mathbb{1}_{A(Q)} \mid \mathcal{G}_{\operatorname{lev} Q}\right), \quad Q \in \mathscr{C}_{\nu}^{(\delta)} .
$$

Combining (5.8) and (5.9), together with Kahane's contraction principle yield

$$
\left\|S_{\lambda}^{(m)} f\right\| \lesssim \int_{0}^{1}\left\|\sum_{Q \in \mathscr{C}_{\nu}^{(\delta)}} r_{Q}(t) f_{Q}\right\| g_{Q, \lambda}^{(m)}\left\|_{\infty} \mathbb{E}\left(\mathbb{1}_{A(Q)} \mid \mathcal{G}_{\operatorname{lev} Q}\right)\right\| \mathrm{d} t
$$

Applying Bourgain's version of Stein's martingale gives

$$
\left\|S_{\lambda}^{(m)} f\right\| \lesssim \int_{0}^{1}\left\|\sum_{Q \in \mathscr{C}_{\nu}^{(\delta)}} r_{Q}(t) f_{Q}\right\| g_{Q, \lambda}^{(m)}\left\|_{\infty} \mathbb{1}_{A(Q)}\right\| \mathrm{d} t .
$$

By (G(1), the support of $g_{Q, \lambda}^{(n)}$ is a subset of $\mathscr{S}_{\lambda}^{(n)}(Q)^{*}$. If we define

$$
V:=\left\{\left|g_{Q, \lambda}^{(n)}\right| \geq \frac{\left\|g_{Q, \lambda}^{(m)}\right\|_{\infty}}{2 C_{g}}\right\} \cap A(Q) \cap \mathscr{S}_{\lambda}^{(n)}(Q)^{*},
$$

then (5.7) and Lemma 5.1 imply

$$
|V| \geq \frac{1}{4 C_{g}^{2}}\left|\mathscr{S}_{\lambda}^{(n)}(Q)^{*}\right| \geq \frac{1}{4 C_{g}^{2}\left(1+K_{1}\right)}|A(Q)| .
$$

As a consequence of the definition of $V$ and (5.11),

$$
\begin{aligned}
\left\|g_{Q, \lambda}^{(m)}\right\|_{\infty} \cdot \mathbb{1}_{A(Q)} & \leq\left(\frac{2 C_{g}}{|V|} \int_{V}\left|g_{Q, \lambda}^{(n)}\right|\right) \cdot \mathbb{1}_{A(Q)} \\
& \leq\left(\frac{8 C_{g}^{3}\left(1+K_{1}\right)}{|A(Q)|} \int_{A(Q)}\left|g_{Q, \lambda}^{(n)}\right|\right) \cdot \mathbb{1}_{A(Q)} \\
& \leq 8 C_{g}^{3}\left(1+K_{1}\right) \cdot \mathbb{E}\left(\left|g_{Q, \lambda}^{(n)}\right| \mid \mathcal{F}_{\operatorname{lev} Q}\right)
\end{aligned}
$$

for all $Q \in C_{\nu}^{(\delta)}$. Plugging the latter estimate into (5.10), Kahane's contraction principle yields

$$
\left\|S_{\lambda}^{(m)} f\right\| \lesssim \int_{0}^{1}\left\|\sum_{Q \in \mathscr{C}_{\nu}^{(\delta)}} r_{Q}(t) f_{Q} \mathbb{E}\left(\left|g_{Q, \lambda}^{(n)}\right| \mid \mathcal{F}_{\operatorname{lev} Q}\right)\right\| \mathrm{d} t .
$$

Subsequently, applying Stein's martingale inequality, Kahane's contraction principle to pass from $\left|g_{Q, \lambda}^{(n)}\right|$ to $g_{Q, \lambda}^{(n)}$ and finally using the UMD-property to dispose of the Rademacher functions, concludes the proof.

Applying the estimate in Theorem 5.3, i.e., the uniform equivalence of stripe operators, we obtain upper and lower estimates for $S_{\lambda}^{(m)}$ via the cotype and type inequalities, respectively. 
Theorem 5.4. Let $X$ be a space of homogeneous type, $E$ a UMD-space and $1<p<\infty$. Moreover, let $\lambda$ be a positive integer and $1 \leq m \leq M$. If we assume

$$
\left\|\sum_{n=1}^{M} S_{\lambda}^{(n)} h_{Q}\right\|_{\infty} \leq C_{S} \cdot \frac{1}{|Q|} \int\left|h_{Q}\right|, \quad Q \in \mathscr{Q}
$$

then

$$
\left\|S_{\lambda}^{(m)} f\right\|_{L_{E}^{p}(X)} \leq C \cdot C_{S} M^{-1 / \mathcal{e}}\|f\|_{L_{E}^{p}(X)}, \quad f \in \overline{\operatorname{span}}\left\{h_{P}: P \in \mathscr{Q}\right\},
$$

where $L_{E}^{p}(X)$ has cotype $\mathcal{C}$ and the constant $C$ depends only on $p, X$ and $E$.

On the other hand, if we assume

$$
\left\|h_{Q}\right\|_{\infty} \leq C_{S} \cdot \sum_{n=1}^{M} \frac{1}{|Q|} \int\left|S_{\lambda}^{(n)} h_{Q}\right|, \quad Q \in \mathscr{Q},
$$

then

$$
\left\|S_{\lambda}^{(m)} f\right\|_{L_{E}^{p}(X)} \geq C \cdot C_{S}^{-1} M^{-1 / \mathcal{T}}\|f\|_{L_{E}^{p}(X)}, \quad f \in \overline{\operatorname{span}}\left\{h_{P}: P \in \mathscr{Q}\right\},
$$

where $L_{E}^{p}(X)$ has type $\mathcal{T}$ and the constant $C$ depends only on $p, X$ and $E$.

Proof. First, we prove inequality (5.13) under the hypothesis (5.12). Let $f=\sum_{Q} f_{Q} h_{Q}$ be a finite sum and $m$ be an integer in the range $1 \leq m \leq M$. By (M2), $\left\{h_{Q}\right\}$ is a martingale difference sequence, thus

$$
\|f\| \gtrsim \int_{0}^{1}\left\|\sum_{Q} r_{Q}(t) f_{Q}\left|h_{Q}\right|\right\| \mathrm{d} t
$$

as a consequence of the UMD-property of $E$ and Kahane's contraction principle. Define the filtration $\left\{\mathcal{F}_{j}\right\}$ by

$$
\mathcal{F}_{j}=\sigma \text {-algebra }\left(\bigcup_{i \leq j} \mathscr{Q}_{i}\right)
$$

Then, Bourgain's version of Stein's martingale inequality yields

$$
\|f\| \gtrsim \int_{0}^{1}\left\|\sum_{Q} r_{Q}(t) f_{Q} \mathbb{E}\left(\left|h_{Q}\right| \mid \mathcal{F}_{\operatorname{lev} Q}\right)\right\| \mathrm{d} t .
$$

Observe that $Q$ is an atom in the $\sigma$-algebra $\mathcal{F}_{\operatorname{lev} Q}$ for all $Q \in \mathscr{Q}$, and thus

$$
\mathbb{E}\left(\left|h_{Q}\right| \mid \mathcal{F}_{\text {lev } Q}\right)=\left(\frac{1}{|Q|} \int\left|h_{Q}\right|\right) \cdot \mathbb{1}_{Q} \geq C_{S}^{-1} \cdot\left\|\sum_{n=1}^{M} S_{\lambda}^{(n)} h_{Q}\right\|_{\infty} \mathbb{1}_{Q},
$$

where we used (M1) and (5.12). Plugging the latter inequality into (5.16), Kahane's contraction principle implies

$$
\|f\| \gtrsim C_{S}^{-1} \int_{0}^{1}\left\|\sum_{Q} r_{Q}(t) f_{Q} \sum_{n=1}^{M} S_{\lambda}^{(n)} h_{Q}\right\| \mathrm{d} t .
$$

Condition (G2) and the UMD-property of $L_{E}^{p}(X)$ yield

$$
\|f\| \gtrsim C_{S}^{-1}\left\|\sum_{j \in \mathbb{Z}} \sum_{n=1}^{M} S_{\lambda}^{(n)}\left(\sum_{Q \in \mathscr{Q}_{j}} f_{Q} h_{Q}\right)\right\| .
$$

Now let

$$
d_{j, n}:=S_{\lambda}^{(n)}\left(\sum_{Q \in \mathscr{Q}_{j}} f_{Q} h_{Q}\right)
$$


and observe that $\left(d_{j, n}\right)$ constitutes a martingale difference sequence with respect to the lexicographic ordering on the index pairs $(j, n)$. Thus, (5.17) implies

$$
\|f\| \gtrsim C_{S}^{-1} \int_{0}^{1}\left\|\sum_{n=1}^{M} r_{n}(t) \sum_{j \in \mathbb{Z}} d_{j, n}\right\| \mathrm{d} t .
$$

Since $L_{E}^{p}(X)$ has cotype $\mathcal{C}$, we employ the cotype inequality to obtain

$$
\|f\| \gtrsim C_{S}^{-1}\left(\sum_{n=1}^{M}\left\|\sum_{j \in \mathbb{Z}} d_{j, n}\right\|^{\mathrm{e}}\right)^{1 / \mathcal{e}}=C_{S}^{-1}\left(\sum_{n=1}^{M}\left\|S_{\lambda}^{(n)} f\right\|^{\mathrm{e}}\right)^{1 / \mathcal{e}} .
$$

By Theorem [5.3, $\left\|S_{\lambda}^{(n)} f\right\| \gtrsim\left\|S_{\lambda}^{(m)} f\right\|$ for all $1 \leq n \leq M$, and therefore

$$
\|f\| \gtrsim C_{S}^{-1} M^{1 / \mathcal{e}}\left\|S_{\lambda}^{(m)} f\right\|
$$

proving (5.13).

A similar argument replacing the cotype inequality by the type inequality proves (5.15) under the condition (5.14).

Acknowledgments. The authors are grateful to P. F. X. Müller for arranging this collaboration and many enlightening discussions. We would like to thank the referee whose suggestions led to the elimination of two technical assumptions originally imposed on the space of homogeneous type. M. P. is supported by the Austrian Science Fund, FWF Project P 23987-N18.

\section{REFERENCES}

[1] S.Y.A. Chang, J.M. Wilson, and T.H. Wolff. Some weighted norm inequalities concerning the Schrödinger operators. Comment. Math. Helv., 60:217-246, 1985.

[2] M. Christ. A $T(b)$ theorem with remarks on analytic capacity and the Cauchy integral. Colloq. Math., 60/61(2):601-628, 1990.

[3] G. David. Wavelets and singular integrals on curves and surfaces, volume 1465 of Lecture Notes in Mathematics. Springer-Verlag, Berlin, 1991.

[4] B. Davis. Hardy spaces and rearrangements. Trans. Amer. Math. Soc., 261(1):211-233, 1980.

[5] T. Figiel. On equivalence of some bases to the Haar system in spaces of vector-valued functions. Bull. Polish Acad. Sci. Math., 36(3-4):119-131 (1989), 1988.

[6] T. Figiel. Singular integral operators: a martingale approach. In Geometry of Banach spaces (Strobl, 1989), volume 158 of London Math. Soc. Lecture Note Ser., pages 95-110. Cambridge Univ. Press, Cambridge, 1990.

[7] J. B. Garnett and P. W. Jones. BMO from dyadic BMO. Pac. J. Math., 99:351-371, 1982.

[8] T. P. Hytönen. Vector-valued singular integrals revisited-with random dyadic cubes. Bull. Pol. Acad. Sci. Math., 60(3):269-283, 2012.

[9] R. Lechner. An interpolatory estimate for the UMD-valued directional haar projection. Diss. Math., to appear.

[10] R. Lechner. The one-third-trick and shift operators. Bull. Pol. Acad. Sci., Math., 61(3-4):219-238, 2013.

[11] J. Lee, P. F. X. Müller, and S. Müller. Compensated Compactness, Separately Convex Functions and Interpolatory Estimates between Riesz Transforms and Haar Projections. Communications in Partial Differential Equations, 36(4):547-601, 2011.

[12] R. A. Macías and C. Segovia. Lipschitz functions on spaces of homogeneous type. Adv. in Math., 33(3):257-270, 1979.

[13] P. F. X. Müller and M. Passenbrunner. A decomposition theorem for singular integral operators on spaces of homogeneous type. J. Funct. Anal., 262(4):1427-1465, 2012.

[14] S. Müller. Rank-one Convexity Implies Quasiconvexity on Diagonal Matrices. International Mathematics Research Notices, 1999(20):1087-1095, 1999.

[15] F. Nazarov, S. Treil, and A. Volberg. Cauchy integral and Calderón-Zygmund operators on nonhomogeneous spaces. Internat. Math. Res. Notices, 15:703-726, 1997. 
[16] F. Nazarov, S. Treil, and A. Volberg. The Tb-theorem on non-homogeneous spaces. Acta Math., 190(2):151-239, 2003.

[17] T. H. Wolff. Two algebras of bounded functions. Duke Math. J., 49:321-328, 1982.

Richard Lechner, Institute of Analysis, Johannes Kepler University Linz, Altenberger Strasse 69, A-4040 Linz, Austria

E-mail address: Richard.Lechner@jku.at

Markus Passenbrunner, Institute of Analysis, Johannes Kepler University Linz, Altenberger Strasse 69, A-4040 Linz, Austria

E-mail address: Markus.Passenbrunner@jku.at 